\title{
Incidence and Impact of Lymph Node Metastases in Advanced Ovarian Cancer: Implications for Surgical Treatment
}

\author{
Cornelia Bachmann ${ }^{\circledR}{ }^{\bowtie}$, Robert Bachmann ${ }^{2}$, Falko Fend ${ }^{3}$, Diethelm Wallwiener ${ }^{1}$ \\ 1. Department of gynecology, University Tübingen, Calwer Str. 7, 72070 Tübingen, Germany; \\ 2. Department of General, Visceral and Transplantation Surgery, University of Tübingen, Hoppe-Seyler Str. 3, 72076 Tübingen, Germany; \\ 3. Department of Pathology, University Tübingen; Liebermeisterstraße 8; 72076Tübingen; Germany. \\ $\triangle$ Corresponding author: Bachmann Cornelia MD. Department of gynecology, University Tübingen, Calwer Str. 7, 72070 Tübingen, Germany. Email: \\ cornelia.bachmann@med.uni-tuebingen.de Phone:0049-7071-2982211 FAX: 0049-7071-294525.
}

(C) Ivyspring International Publisher. Reproduction is permitted for personal, noncommercial use, provided that the article is in whole, unmodified, and properly cited. See http://ivyspring.com/terms for terms and conditions.

Received: 2016.03.25; Accepted: 2016.09.18; Published: 2016.11.09

\begin{abstract}
Background: This study aimed to clarify the impact of node involvement (affected to resected nodes) in optimally cytoreduced (residual tumour $\leq 1 \mathrm{~cm}$ ) stage IIIC/IV ovarian cancer.

Methods: 108 consecutive patients with primary stage IIIC/IV ovarian cancer underwent stage-related surgery and got adjuvant platinum-based chemotherapy. Median follow-up: 53.5 months. All patients got systematic para-aortic and pelvic lymphadenectomy. Clinical parameters were retrospectively evaluated. Patients were stratified into 3 groups to evaluate node affection: 1) no $(0 \%), 2)$ minor ( $>0 \%, \leq 50 \%)>0$ and 3 ) major (>50\% of affected nodes). Kaplan-Meier survival curve was used to evaluate the prognostic value.

Results: On average, 21.3 pelvic and para-aortic nodes were removed per patient (range 1-60 nodes). Minor nodal involvement (node ratio >0- $\leq 0.5$ : (59\%) was most often detected. Increasing node ratio leads to significant decreased overall survival $(p<0.001)$. Significant best overall survival was associated with minor node involvement (node ratio $>0$ to $\leq 0.5$ ). Complete cytoreduction correlated with node affection shows significant best prognostic impact in minor node affection compared to incomplete resection $(R>0-\leq 1 \mathrm{~cm})$ independent to nodal status (OS $p<0.001$ ).

Conclusion: Radical surgery is the main factor of improved overall and tumor free survival. Paraaortal and iliacal lymphadenectomy seems to play an important role for prognostic and therapeutic reasons: Prognostic in accurate staging and therapeutic in case of achieved optimal cytoreduction including lymph nodes with histology proven minor node involvement.
\end{abstract}

Key words: Advanced ovarian cancer; node ratio; lymphadenectomy; prognosis; residual tumour; node involvement.

\section{Introduction}

The initial management of primary ovarian cancer includes surgical staging, cytoreductive surgery, lymphadenectomy followed by a platinumbased chemotherapy, except for pT1aG1 cases [1,2]. However, the importance of systematic lymphadenectomy in primary advanced ovarian cancer and its prognostic relevance is still unclear $[3,4,5]$. The increasing node involvement in advanced ovarian cancer is known with unidentified prognostic impact [6,7]. A rate of about $50 \%$ of node metastases has been observed $[1,8]$ and accurate surgical staging, including lymphadenectomy, recognizes the true extent of disease by detection of occult node metastases. Many studies have reported a better prognosis for stage IIIC ovarian cancer patients with sole lymph node metastases (without peritoneal 
carcinomatosis) compared to lymph node metastases and concomitant peritoneal carcinomatosis [9]. The new FIGO classification is considering this fact [10]. Our objective was to delineate the incidence and impact of pelvic and paraaortic node metastases in optimally cytoreduced $(\mathrm{R} \leq 1 \mathrm{~cm})$ stage IIIC/IV ovarian cancer patients.

\section{Material/ Methods}

A total of 108 consecutive patients with primary stage IIIc/IV (according to FIGO) optimally cytoreduced $(\mathrm{R} \leq 1 \mathrm{~cm})$ ovarian cancer were enrolled. Each patient underwent surgical staging followed by hysterectomy, bilateral adnexectomy omentectomy, pelvic and para- aortic lymphadenectomy or tumour debulking as clinically indicated. Pelvic and para-aortic lymphadenectomy was performed up to the level of the renal vessels in all patients with optimally cytoreduced ovarian cancer $(\mathrm{R} \leq 1 \mathrm{~cm})$ and in good state of health (Karnofsky- Index $\geq 80 \%$ ); this procedure was performed in every case. All of the patients were treated with an adjuvant standard platinum- based chemotherapy.

Optimal cytoreduction is defined as a residual tumour mass $\leq 1 \mathrm{~cm}$ and complete cytoreduction is defined as a residual tumour mass $=0 \mathrm{~mm}$. Patients with suboptimal cytoreduction $(R>1 \quad \mathrm{~cm})$ were excluded. All patients were evaluated with respect to age at diagnosis, stage, histology, histologic grade and residual tumour mass. Patients' characteristics are given in table 1 . On average, 21.3 pelvic and para-aortic lymph nodes were removed per patient (range 1 - 60 nodes). 108 patients met the inclusion criteria and were further evaluated. In median, the age of all patients was 60.2 years (range 25- 83 y). Every patient gave written informed consent for data acquisition prior to their inclusion in study. All surgical pathologic samples were examined by a gynecological pathologist. The histological diagnosis was classified according to FIGO- stages [11]. Residual tumor mass was subdivided in the following groups: $\mathrm{R} 0=$ complete cytoreduction $(=0 \mathrm{~mm})$ and $\mathrm{R}>0 \mathrm{~mm}-\leq 10 \mathrm{~mm}$.

For the evaluation of the prognostic impact of lymph node metastases the patients were stratified into 3 groups depending on the extent of node involvement (node ratio=NR (affected to removed nodes) depending on no, minor and major nodal involvement: 1 . no lymphnode metastases $(\mathrm{NR}=0)$; 2 . $>0$ and less than $50 \%$ of involved nodes (NR $>0-\leq 0.5$ ); 3. more than $50 \%$ of involved nodes (NR: $>0.5-\leq 1$ ).

\section{Follow up}

Follow up- data were collected when the patients presented in our department for follow up. The mean follow-up time was 53.5 months. Follow- up data of all 108 patients were evaluated.

For evaluation of the OS and PFS data on one patient were missing and this patient was not further evaluated in this subgroup. OS and PFS were evaluated of 107 patients.

Table 1. Patients' characteristics. 108 patients in FIGO IIIC/ IV were analysed. Node involvement was analysed: for evaluation of lymph node ratio (affected to removed nodes) patients are stratified into 3 groups: $0 ;>0-\leq 0.5$ and $>0.5-\leq 1$ (see materials and methods).

\begin{tabular}{ll}
\hline Parameter & $\mathrm{N}(\%)$ \\
\hline FIGO IIIC/IV & $108(100)$ \\
Histologic grade & \\
G1/2 & $48(44.44)$ \\
G3 & $60(55.55)$ \\
Histology & \\
Serous & $94(87.04)$ \\
Non Serous & $14(12.96)$ \\
R-Status & \\
R $=0 \mathrm{~mm}$ & $39(36.45)$ \\
R $>0 \mathrm{~mm}-\leq 1 \mathrm{~cm}$ & $68(63.55)$ \\
N-Status & \\
N0 & $27(25.0)$ \\
N+ & $81(75.0)$ \\
Lymph Node ratio (affected to removed nodes) & \\
0 & $27(25.0)$ \\
$>0-\leq 0.5$ & $64(59.26)$ \\
$>0.5-\leq 1$ & $17(15.74)$ \\
\hline
\end{tabular}

\section{Statistical analysis}

Data were stored in a database and analyzed using PASW (Version 22 SPSS Inc., Chicago, IL., USA). Univariate analyses were performed using PASW (Version 22 SPSS Inc., Chicago, IL., USA). The results are expressed as means, standard deviations, minimums, maximums and percentages. KaplanMeier analyses were used to calculate hazard ratio and 95\% CI for OS/ PFS. The log- rank test was used to test for significant differences between the groups. P-values $<0.05$ were considered statistically significant.

\section{Results}

Of the enrolled patients, all got optimal cytoreduction: $63.55 \%$ had $>0 \mathrm{~mm}$ and $\leq 10 \mathrm{~mm}$ residual tumour mass and $36.45 \%$ had complete cytoreduction, respectively (table 1). The patients' characteristics are summarised in Table 1. Overall, most often node metastases $(75.0 \%)$, histological grade $3(55.55 \%)$ and serous histology $(87.04 \%)$ were detected (table 1). A lymph node ratio between $>0$ to $\leq 0.5$ mainly occurs, $59.26 \%$ of the patients had $>0$ and less than $50 \%$ of affected nodes (table 1). $25 \%$ of the 
patients had no node metastases (node ratio $=0$; table $1)$; least frequently a node involvement of $>50 \%$ (node ratio $>0.5-1$ ) was seen in about $15.74 \%$ (table 1 ).

Concerning the impact of clinicopathologic parameters on node metastases the following was observed (table 2): most often a node ratio $>0$ - $\leq 0.5$ was associated with histologic grade 3 , serous cancers and residual tumour mass $>0 \mathrm{~mm}-1 \mathrm{~cm}$ (table 2 ). Much rarer, a node ratio $>0.5$ regardless of histologic grade, histology and residual tumour mass was detected (table 2).

Table 2. Relations between lymph node involvement (node ratio) and the clinicopathological parameters (histologic grade, histological subtypes, R-status) in FIGO IIIc/IV; $n=108$.

\begin{tabular}{llll}
\hline & \multicolumn{2}{l}{ Node ratio $\mathrm{n}(\%)$} & \\
\cline { 2 - 4 } Parameter & 0 & $>0-\leq 0.5$ & $>0.5-\leq 1$ \\
\hline Histologic grade & & & \\
G1 $/ 2$ & $15(13.88)$ & $27(25.0)$ & $6(5.55)$ \\
G3 & $12(11.11)$ & $37(34.26)$ & $11(10.19)$ \\
Histology & & & \\
Serous & $24(22.22)$ & $57(52.77)$ & $13(12.04)$ \\
Non Serous & $3(2.77)$ & $7(6.48)$ & $4(3.7)$ \\
R- Status & & & \\
R= 0mm & $11(10.19)$ & $23(21.3)$ & $5(4.63)$ \\
R $>0-\leq 1 \mathrm{~cm}$ & $16(14.81)$ & $41(38.0)$ & $12(11.11)$ \\
\hline
\end{tabular}

The significant best prognostic impact on OS and PFS have patients with complete cytoreduction compared to $\mathrm{R}>0 \mathrm{~mm}-\leq 1 \mathrm{~cm}$, respectively. Hereafter the prognostic impact of the lymph node involvement (node ratio) on OS and PFS in optimally cytoreduced patients was investigated (table 3): major nodal involvement ( $>50 \%$ affected lymph nodes) showed strong influence on OS and was associated with significant decreased survival (OS), respectively, (table 3). Thus, a significant prognostic advantage on OS was seen for patients with complete cytoreduction and moderate (minor) lymph node involvement ( $>0$ to $\leq 0.5 ; \mathrm{p}<0.001$, table 3) compared to the other groups (table 3).

Subsequently, even PFS is significantly influenced by an increasing node affection $(\mathrm{p}<0.006$; table 3). Patients with a moderate lymph node involvement showed longer PFS ( $>0$ to $\leq 0.5$; table 3 ) compared to the other 2 groups (table 3 ). Strong lymph node involvement (node ratio $>0.5-\leq 1$ ) showed worst prognosis in optimally cytoreduced patients (OS/PFS; table 3). Subsequently, OS is significantly influenced by node affection $(p<0.001$; table 4) and a complete cytoreduction compared to residual tumour $>0 \mathrm{~mm}-10 \mathrm{~mm}$ independent to nodal involvement. Patients with a moderate lymph node involvement and complete cytoreduction showed longer OS ( $>0$ to $\leq 0.5$; table 4 ) compared to the other 2 groups (table 4). Strong lymph node involvement (node ratio $>0.5-\leq 1$ ) showed worst prognosis in optimally cytoreduced patients (OS/PFS; table 4). The prognostic impact on PFS is significant influenced by moderate lymph node involvement and complete cytoreduction $(\mathrm{p}=0.017$; table 4$)$.

Table 3: Prognostic impact of lymph node involvement on over-all survival and progression-free survival (OS/ PFS; months, $95 \% \mathrm{Cl}$ ) in 107 stage IIIc/IV patients after optimal cytoreduction (R. $\leq 1 \mathrm{~cm})$.

\begin{tabular}{lllll}
\hline Parameter & $\begin{array}{l}\text { PFS (months; } \\
95 \% \text { CI) }\end{array}$ & p-value & $\begin{array}{l}\text { OS (months; 95\% } \\
\text { CI) }\end{array}$ & p-value \\
\hline $\begin{array}{l}\text { Node ratio } \\
0(\mathrm{n}=26)\end{array}$ & $13.7(11.7-15.8)$ & & $24.5(20.58-28.36)$ & \\
$>0-\leq 0.5(\mathrm{n}=64)$ & $14.9(11.02-18.8)$ & 0.006 & $30.5(24.7-57.3)$ & $\mathrm{P}<0.001$ \\
$>0.5(\mathrm{n}=17)$ & $10.2(9.2-11.2)$ & & $18.8(9.7-27.9)$ & \\
\hline
\end{tabular}

Table 4: Prognostic impact of lymph node involvement on over-all survival and progression-free survival (OS/ PFS; months, $95 \% \mathrm{Cl}$ ) in 107 in stage IIIc/IV patients depending on residual tumour mass $(R=0 \mathrm{~mm}$ and $\mathrm{R}>0-1 \mathrm{~cm})$.

\begin{tabular}{lllll}
\hline Parameter & $\begin{array}{l}\text { PFS (months; } \\
95 \% \text { CI })\end{array}$ & p- value & $\begin{array}{l}\text { OS (months; } \\
95 \% \mathrm{CI})\end{array}$ & p- value \\
\hline $\begin{array}{l}\mathrm{R}=0 \mathrm{~mm} \text { Node ratio } \\
0(\mathrm{n}=11)\end{array}$ & $17.5(0-48.21)$ & & $36.3(0-68.6)$ & \\
$>0-\leq 0.5(\mathrm{n}=23)$ & $29.5(18.5-40.4)$ & & $50.5(41.5-58.1)$ & \\
$>0.5(\mathrm{n}=5)$ & $12.17(1.8-22.5)$ & & $12.8(0.7-24.9)$ & \\
$\mathrm{R}>0 \mathrm{~mm}-1 \mathrm{~cm}$ Node & & 0.017 & & $<0.001$ \\
ratio & & & & \\
$0(\mathrm{n}=15)$ & $12.6(8.0-17.2)$ & & 24.5 & $(21.25-27.7)$ \\
& & & $27.9(22.6-33.1)$ & \\
$>0-\leq 0.5(\mathrm{n}=41)$ & $13.2(11.7-14.7)$ & & $18.8(11.2-26.4)$ & \\
$>0.5(\mathrm{n}=12)$ & $10.1(9.2-11.04)$ & &
\end{tabular}

\section{Discussion}

The optimal cytoreduction is the known significant most important prognostic factor in advanced ovarian cancer $[8,9,12,13,14]$. Complete cytoreduction leads to significantly better prognosis than cytoreduction $>0 \mathrm{~mm}$ to $10 \mathrm{~mm}$ [15]. Further known significant prognostic factors are FIGO stage, histology and histologic grade $[15,16]$.

The prognostic relevance of a lymphadenectomy in the surgical management of ovarian cancer is still unclear $[13,17,18]$ and is currently investigated in the prospective LION study (AGO-Ovar). Results of randomized controlled studies are still missing $[19,20]$, but might probably answer the therapeutic 
and prognostic impact of lymphadenectomy in initial management of ovarian cancer in a greater collective. In primary ovarian cancer, a pelvic and paraaortic lymphadenectomy after optimal cytoreduction is recommended with positive prognostic effect $[19,21]$. The randomized trial of Panici showed a positive impact of a systemic lymphadenectomy on PFS compared to resection of bulky nodes, but no impact on overall survival (OS) in optimally cytoreduced patients $[3,4,22]$. In contrast, Pereira showed a positive prognostic impact with a systematic lymphadenectomy with a significant longer survival in advanced ovarian cancer [4].

In advanced ovarian cancer node metastases are known in about $40 \%$, even with affection of the pelvic and/ or para- aortic region $[3,22]$. In our collective node metastases were detected in $75.0 \%$ (table 1 ). Additionally, our data detected an association of node metastases most often with serous cancers, histologic grade 3 and residual tumour mass $>0-1 \mathrm{~cm}$ (table 2); most often a moderate lymph node involvement was detected ( $>0-\leq 0.5$; table 2$)$. Most of these results are similar to previous reports, but to our knowledge, the extent of node involvement (node ratio (affected to removed)) was rarely included in other reports of risk factors for ovarian cancer before.

The prognostic relevance of node metastases in primary ovarian cancer is still unclear $[4,23]$. One study reported that the influence of lymph node metastases on prognosis decreases with the increase of residual tumor mass $[6,9,24,25]$. The authors also reported that node metastases seemed to be the second most important prognostic factor for advanced-stage ovarian cancer [6]. Although many risk factors of ovarian cancer are known, it is still questionable if lymphadenectomy in advanced ovarian cancer improves prognosis.

Unquestionable is that complete cytoreduction compared to $\mathrm{R}>0 \mathrm{~mm}-\leq 1 \mathrm{~cm}$ has significant best prognostic impact (PFS/ OS) $[8,12,13,14]$; even seen in our study (table 3). Out of our date the combination of radical surgery with lymphadeectomy seems additionally favourable (Table 4). The prognostic impact of clinicopathological factors associated with the node ratio has to be investigated in larger studies to improve the prognostic relevance of node metastases in FIGO IIIC. Mahdi [23] described that the impact of increasing node ratio was strongly related to OS, especially in patients with no macroscopic peritoneal disease [23]. Our study showed similar results; a node ratio $(>0.5)$ was associated with significantly decreased survival, respectively $(\mathrm{p}<0.001$; table 3$)$. Patients with node positive ovarian cancer of less than $50 \%$ of removed nodes (ratio: $>0$ to $\leq 0.5$ ) have an improved OS with significant positive prognostic impact (table 3). Significant best impact on OS was seen with decreasing node ratio, especially for patients with less than $50 \%$ of affected nodes ( $<<0.001$; $>0$ to $\leq 0.5$; table 3 ). Strong lymph node involvement $(>0.5-\leq 1)$ had worst prognosis (table 3 ), so affection of nodes seems to play a role in the prognosis in optimally cytoreduced patients.

Possibly in our study the group with a ratio $>0$ to $\leq 0.5$ could contain patients with sole lymph node involvement without peritoneal lesions and are staged up to FIGO IIIC/IIIA1 [22,26,27]. These group of patients showed significant best OS in our study (table 4), as described in one report that ovarian serous carcinoma patients with sole extrapelvic peritoneal involvement have better survival than those with extrapelvic peritoneal involvement and lymph node metastases [11]. Additionally, an adequate staging is possible by performing a lymphadenectomy [5]. Considering the relatively favorable prognosis associated with lymphatic tumor spread compared with peritoneal tumor spread (stage IIIC), which was classified solely on the basis of lymph node metastasis, SU et al. suggests a modified FIGO classification with a down-staging of these patients [9]. Berek argued that FIGO should consider modifying the ovarian cancer staging by further stratifying stage III disease on the basis of the better OS in patients with retroperitoneal node metastasis without peritoneal carcinomatosis than in patients with macroscopic peritoneal carcinomatosis. One plausible explanation for the favorable prognosis of those patients might be the higher rate of optimal cytoreduction compared to the patients with stage IIIC disease showing intraperitoneal tumor implants $>2 \mathrm{~cm}$ [9]. Our data support this hypothesis. Meanwhile there does exist a new classification for ovarian cancer in which this fact is considered [10]. Since ovarian cancer is known to spread simultaneously both intraperitoneally and retroperitoneally, the presence of tumor spreading mainly through lymphatic channels without intraperitoneal dissemination suggests that such tumors might be associated with a favorable biologic behavior [9].

Potentially these patients with minor node involvement could benefit from a systematic lymphadenectomy in FIGO IIIC compared to patients with strong node involvement (node ratio: $>0.5-1$ ). If the prognostic impact is caused by removing of positive nodes is still unclear, but maybe these results can help in treatment decisions.

Perhaps the stratification of this subpopulation of node positive EOC based on nodal burden provides a significant prognostic value that may be considered in future staging and aid in management decisions 
[23]; our study supports this hypothesis: an increasing node involvement leads to worse prognosis.

In our study, interesting results were found and node ratio might be prognostically interesting. Based on our survey, node ratio may be used to 1) guide intraoperative decision making regarding lymphadenectomy in incomplete cytoreduction with possible abandonment of lymphadenectomy and 2) to estimate the prognosis (OS) in patients with advanced ovarian cancer after optimal cytoreduction. The outstanding results from the current prospective LION study (AGO-Ovar) will help to answer the validity of the lymphadenectomy on treatment strategies in optimally cytoreduced patients.

\section{Conclusion}

Main intention of primary surgery in advanced ovarian cancer is optimal cytoreduction with significant best prognostic impact. More extensive lymphadenectomy seems to play an important role in providing accurate staging and the node ratio might give prognostic information in optimally cytoreduced stage IIIc/IV ovarian cancer. The modification of the FIGO staging system, done in 2014, especially for stage IIIC ovarian cancer patients, has considered the prognostic differences depending on nodal involvement and complete cytoreduction. These changes are in the line with our results leading to downstaging (FIGO IIIC to FIGO IIIA1) of patients with exclusive nodal involvement with precisely best prognostic impact in our collective.

\section{Acknowledgments}

We acknowledge support by Deutsche Forschungsgemeinschaft and Open Access Publishing Fund of University of Tübingen.

\section{Ethical standards}

Our investigation of 108 patients has been approved by the appropriate ethics committee and has therefore been performed in accordance with the ethical standards laid down in the 1964 Declaration of Helsinki. All persons gave their informed consent prior to their inclusion in the study.

\section{Competing Interests}

The authors have declared that no competing interest exists.

\section{References}

[1] Du Bois A, et al. Role of surgical outcome as prognostic factor in advanced epithelial ovarian cancer: a combined exploratory analysis of 3 prospectively randomized phase 3 multicenter trials. Cancer 2009:: 1234-44

[2] Bristow RE, Tomacruz RS et al. Survival effect of maximal cytoreductive surgery for advanced ovarian carcinoma during the platinum era: a meta-analysis. J Clin Oncol. 2002 Mar 1;20(5):1248-59.
[3] Panici PB, Maggioni A et al. Systematic aortic and pelvic lymphadenectomy versus resection of bulky nodes only in optimally debulked advanced ovarian cancer: a randomized clinical trial. J Natl Cancer Inst. 2005 Apr 20;97(8):560-6.

[4] Pereira A, Pérez-Medina T, Magrina JF, Magtibay PM, Millan I, Iglesias E. The role of lymphadenectomy in node-positive epithelial ovarian cancer. Int J Gynecol Cancer. 2012 Jul;22(6):987-92.

[5] Scarabelli C, Gallo A, Visentin MC et al. Systematic pelvic and para-aortic lymphadenectomy in advanced ovarian cancer patients with no residual intraperitoneal disease. Int J Gynecol Cancer. 1997 Jan;7(1):18-26.

[6] Bachmann C, Bachmann S, Fehm T, Staebler A, Becker S, Rothmund R, Gardanis C, Grischke EM, Wallwiener D, Solomayer EF. Nodal status--its impact on prognosis in advanced ovarian cancer. J Cancer Res Clin Oncol. 2012 Feb;138(2):261-7

[7] Harter P1, Gnauert K, Hils R et al. Pattern and clinical predictors of lymph node metastases in epithelial ovarian cancer. Int J Gynecol Cancer. 2007;17(6):1238-44. Epub 2007 Apr 12.

[8] Wimberger P, Lehmann N, Kimmig R, Burges A, Meier W, Du Bois A; Arbeitsgemeinschaft Gynaekologische Onkologie Ovarian Cancer Study Group. Prognostic factors for complete debulking in advanced ovarian cancer and its impact on survival. An exploratory analysis of a prospectively randomized phase III study of the Arbeitsgemeinschaft Gynaekologische Onkologie Ovarian Cancer Study Group (AGO-OVAR). Gynecol Oncol 2007 Jul ; 106(1): 69-74.

[9] Suh DH, et al. Improvements to the FIGO staging for ovarian cancer: reconsideration of lymphatic spread and intraoperative tumor rupture. J Gynecol Oncol. 2013; 24(4): 352-358.

[10] Höhn AK, Einenkel J, Wittekind C, Horn LC. New FIGO classification of ovarian, fallopian tube and primary peritoneal cancer. Pathologe. 2014 Jul;35(4):322-6. doi: 10.1007/s00292-014-1908-0. German.

[11] Bakkar R, Gershenson D, Fox P, Vu K, Zenali M, Silva E. Stage IIIC Ovarian/Peritoneal Serous Carcinoma: A Heterogeneous Group of Patients With Different Prognoses. Int J Gynecol Pathol. 2014 May;33(3):302-8. doi: 10.1097/PGP.0b013e3182988dfd.

[12] Abe A, Furumoto H, Irahara M, Ino H, Kamada M, Naka O, Sasaki M, Kagawa T, Okitsu O, Kushiki N. The impact of systematic para-aortic and pelvic lymphadenectomy on survival in patients with optimally debulked ovarian cancer. J Obstet Gynaecol Res. 2010 Oct;36(5):1023-30.

[13] Nomura H, Tsuda H, Susumu N, Fujii T, Banno K, Kataoka F, Tominaga E, Suzuki A, Chiyoda T, Aoki D. Lymph node metastasis in grossly apparent stages I and II epithelial ovarian cancer. J Surg Oncol. 2011 Jan 16.

[14] Chekerov R, Braicu I, Castillo-Tong DC, Richter R, Cadron I, Mahner S, Woelber L, Marth C, Van Gorp T, Speiser P, Zeillinger R, Vergote I, Sehouli J. Outcome and clinical management of 275 patients with advanced ovarian cancer International Federation of Obstetrics and Gynecology II to IV inside the European Ovarian Cancer Translational Research Consortium-OVCAD. Int J Gynecol Cancer. 2013 Feb;23(2):268-75

[15] Hoskins, McGuire et al. The effect of diameter of largest residual disease on survival after primary cytoreductive surgery in patients with suboptimal residual epithelial ovarian carcinoma. Am J Obstet Gynecol. 1994 Apr;170(4):974-9;

[16] Ulker V, Kuru O, Numanoglu C, Akbayır O, Polat I, Uhri M. Lymph node metastasis in patients with epithelial ovarian cancer macroscopically confined to the ovary: review of a single-institution experience. Arch Gynecol Obstet. 2014 May;289(5):1087-92. doi: 10.1007/s00404-013-3078-3. Epub 2013 Nov 9

[17] di Re F, Baiocchi G, Fontanelli R, Grosso G, Cobellis L, Raspagliesi F, di Re E. Systematic pelvic and paraaortic lymphadenectomy for advanced ovarian cancer: prognostic significance of node metastases. Gynecol Oncol $1996 \mathrm{Sep}$; 62(3): $360-5$

[18] Aletti GD1, Dowdy S, Podratz KC et al. Role of lymphadenectomy in the management of grossly apparent advanced stage epithelial ovarian cancer. Am J Obstet Gynecol. 2006 Dec;195(6):1862-8.

[19] Carnino, Fuda et al. Significance of lymph node sampling in epithelial carcinoma of the ovary. GynecolOncol 1997 Jun;65(3):467-72.

[20] Kim HS, Ju W, Jee BC et al. Systematic lymphadenectomy for survival in epithelial ovarian cancer. Int J Gynecol Cancer. 2010 May;20(4):520-8. doi: 10.1111/IGC.0b013e3181d6de1d.

[21] Onda $T$, Yoshikawa $H$, Yasugi $T$ et al. Patients with ovarian carcinoma upstaged to stage III after systematic lymphadenctomy have similar survival to Stage I/II patients and superior survival to other Stage III patients. Cancer. 1998 Oct 15;83(8):1555-60.

[22] Takeshima $\mathrm{N}$ et al. Lymph node metastasis in ovarian cancer: difference between serous and non-serous primary tumours. Gynecol Oncol. 2005 Nov;99(2):427-31

[23] Mahdi H, Thrall M, Kumar S, Hanna R, Seward S, Lockhart D, Morris RT, Swensen R, Munkarah AR. The prognostic impact of the ratio of positive lymph nodes on survival of epithelial ovarian cancer patients. J Surg Oncol. 2011 Jun 1:103(7):724-9.

[24] Chang SJ, Bristow RE, Ryu HS. Prognostic significance of systematic lymphadenectomy as part of primary debulking surgery in patients with advanced ovarian cancer. Gynecol Oncol. 2012 Sep;126(3):381-6.

[25] Balbi G, Manganaro MA, Monteverde A, Landino I, Franzese C, Gioia F. Ovarian cancer: lymph node metastases. Eur J Gynaecol Oncol. 2009;30(3):289-91. 
[26] Chan JK1, Urban R, Hu JM et al. The potential therapeutic role of lymph node resection in epithelial ovarian cancer: a study of 13918 patients. Br J Cancer. 2007 Jun 18;96(12):1817-22. Epub 2007 May 22.

[27] Denny L, Quinn M, Hacker N. FIGO Cancer Report 2012. Int J Gynaecol Obstet. 2012 Oct; 119 (Suppl 2):S89. 